\title{
Global Perspectives: The COVID-19 Pandemic in 2020-2021 and Beyond
}

\author{
Youngmee Jee
}

While coronavirus disease 2019 (COVID-19) cases have reached 100 million across the globe with more than 2 million deaths as at January 26, 2021, over 50 countries have initiated vaccinations against the disease. Vaccinations began within a year from January 12, 2020, when the China CDC shared the new virus genomic sequence with the World Health Organization (WHO) and globally, following the first complete genome sequencing of the novel $\beta$ genus coronavirus on January 3 (1). This must be the most remarkable achievement in vaccine research and development since the English physician Edward Jenner pioneered the concept of smallpox vaccination in the 18th century. It truly is an outcome of joint efforts between interconnected scientific communities, the leading roles played by the WHO, Global Alliance for Vaccines and Immunisation (GAVI), and Coalition for Epidemic Preparedness Innovations (CEPI), and the contributions of many global health partners.

In 2015, in the aftermaths of the Ebola and Middle East respiratory syndrome (MERS) outbreaks, the WHO created a dedicated research team under the name "the R\&D Blueprint to Prevent Epidemics." The intended aim was to facilitate research and development activity during the time of any outbreak. The R\&D Blueprint organized the First Global Research and Innovation Forum on February 11-12, 2020, less than a fortnight after the declaration of a Public Health Emergency of International Concern (PHEIC) by the WHO Director General, which happened on January 30, 2020. This testifies to the WHO's strong commitment to play a coordinator role in research and development. The First Forum resulted in a coordinated global research roadmap for COVID19, which was published on March 12, 2020. This roadmap proposes an immediate goal of accelerating research to contain the COVID-19 epidemic and a mid- to long-term goal of supporting research priorities that can lead to the development of a global research platform to better prepare for future epidemics and pandemics. There is no doubt that the research and development preparedness coordinated by the WHO
R\&D Blueprint network has facilitated the development of COVID-19 vaccines in 2020.

To end the COVID-19 pandemic, we need an equitable distribution of safe and effective vaccines on a global scale. The WHO, GAVI, and CEPI launched COVAX with a view to ensuring global equitable access to COVID-19 vaccines. However, COVAX's aim of delivering vaccine doses to at least $20 \%$ of the population of each participating country, regardless of the country's income level, as early as possible seems to be difficult to achieve given the foreseen insufficiency of global supply in 2021. At the 148th session of the WHO Executive Board Meeting on January 18, 2021, the WHO Director General made a statement emphasizing the importance of equitable distribution. He called on all countries to work together in solidarity to ensure that, within the first 100 days of 2021, vaccination of health workers and older people is underway in all countries, and requested vaccine producers to prioritize supplying to COVAX rather than through bilateral deals with countries (2). GAVI's forecast that as many as 1.8 billion doses will be available to 92 lower-middle and low-income economies participating in COVAX can only come true when the COVAX Advanced Market Commitment (AMC) proves to be fully functional.

There are still many unknowns in the newly developed COVID-19 vaccines - inter alia the duration and characteristics of vaccine-induced immunity, longterm safety, and efficacy against COVID-19 virus variants. Recent reports of Phase 3 clinical trials from Johnson \& Johnson and Novavax show a reduced efficacy for UK and South African variants (3-4). With expert discussions underway, vaccine manufacturers need to be prepared for the reformulation of vaccines against the currently circulating and upcoming variant strains.

While we need to confirm the efficacy and longterm safety of vaccines that are available and will be available, it can take at least some months to see any decreasing trend of confirmed cases even in countries that have begun vaccination. Furthermore, we may not 
be able to go back to the lifestyles of the pre-COVID19 era even after herd immunity for COVID-19 is achieved. It will take some years to have answers for many unknowns in COVID-19 science. Researchers from academia, industries, governments, nongovernmental organizations (NGOs), and public health partners will have to work together to find those answers. This pandemic has brought us many formidable challenges, but has also provided a precious opportunity to advance science and global cooperation.

doi: $10.46234 / \mathrm{ccdcw} 2021.040$

\# Corresponding author: Youngmee Jee, youngmee.jee@ip-korea.org.

Submitted: January 31, 2021; Accepted: February 01, 2021

\section{REFERENCES}

1. Tan WJ, Zhao X, Ma XJ, Wang WL, Niu PH, Xu WB, et al. Notes from the field: a novel coronavirus genome identified in a cluster of pneumonia cases-Wuhan, China 2019-2020. China CDC Wkly 2020;2(4):61 - 2. http://dx.doi.org/10.46234/ccdcw2020.017.

2. World Health Organization. WHO Director-General's opening remarks at 148th session of the Executive Board. https://www.who.int/directorgeneral/speeches/detail/who-director-general-s-opening-remarks-at148th-session-of-the-executive-board. [2021-1-29].

3. Johnson \& Johnson. Johnson \& Johnson announces single-shot Janssen COVID-19 vaccine candidate met primary endpoints in interim analysis of its phase 3 ENSEMBLE trial. https://www.jnj.com/johnson-johnsonannounces-single-shot-janssen-covid-19-vaccine-candidate-met-primaryendpoints-in-interim-analysis-of-its-phase-3-ensemble-trial. [2021-1-29].

4. Novavax. Novavax COVID-19 vaccine demonstrates $89.3 \%$ efficacy in UK phase 3 trial. https://ir.novavax.com/news-releases/news-releasedetails/novavax-covid-19-vaccine-demonstrates-893-efficacy-uk-phase-3. [2021-1-29].

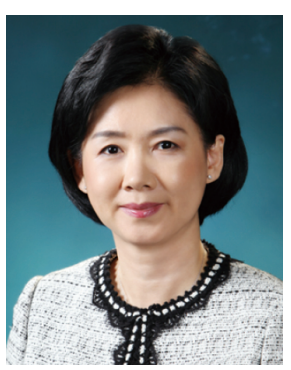

Youngmee Jee, MD, PhD

Chief Executive Officer, Institut Pasteur Korea

Member of the WHO COVID-19 IHR Emergency Committee and the WHO R\&D Blueprint Scientific Advisory Group 